\title{
Trade-off Between Strength and Ductility of Random Discontinuous Fiber Reinforced Cementitious Composites
}

\author{
Hwai-Chung Wu \& Victor C. Li \\ Advanced Civil Engineering Materials Research Laboratory, Department of Civil and Environmental Engineering, \\ University of Michigan, Ann Arbor, Michigan, 48109-2125 USA
}

(Received 8 July 1993; accepted 30 August 1993)

\begin{abstract}
It is well known that fiber reinforcement is an effective way of improving toughness of brittle materials. However, it is less clear how fiber reinforcement (particularly for random discontinuous fibers) can contribute to strength and ductility enhancement of the composites. In this paper, we address this issue from a micromechanics point of view. The conditions for multiple cracking, which is the mechanism of pseudo ductility, are briefly reviewed in terms of micromechanical parameters such as fiber aspect ratio, matrix toughness, and interfacial bond strength. Special attention is given to examining competing effect of these microparameters on composite strength and ductility. Experimental results of direct tensile tests of several random discontinuous fiber-reinforced high strength concretes and OPC paste are presented and discussed in light of recent advancements in pseudo strain-hardening theory.
\end{abstract}

Keywords: Fiber reinforcement, tensile strength, pseudo-strain-hardening, micromechanics, composite design.

\section{INTRODUCTION}

Cement and concrete are most commonly used as construction materials. Although modern Portland cement was discovered in the nineteenth century, its ancient history can be traced back several thousand years.' These materials are mainly used to carry compressive loads due to their brittleness; for tension members reinforcing bars are frequently incorporated in the structural design for carrying tensile loads. However, matrix cracking and spalling often lead to exposure of steel bars. Aggressive agents such as water and chloride ion easily migrate and attack steel reinforcement, which causes further matrix cracking and spalling. Eventually, the integrity of structures is lost. It has been estimated that the rehabilitation cost in the US will reach trillions of dollars over the next twenty years. ${ }^{2}$ In addition to property loss, human life is also frequently threatened as exemplified in the tragic collapse of a California highway bridge in the 1989 Loma Prieta earthquake. The reinforced concrete columns and connectors could have survived with a concrete of a high ductility.

In the past decades, a great deal of effort has been expended on improving the strength (particularly compressive strength) of cementitious materials. This is achieved through (1) better understanding of hydration chemistry, hence optimum chemical composition and curing conditions; (2) graded particle systems, hence more dense packing; and (3) special processing, hence reduced porosity.

In practice, combinations of the above-mentioned strategies have been applied in production of high-strength concrete. Among them, chemically bonded ceramics (CBCs), ${ }^{3,4}$ densified systems containing homogeneously arranged ultrafine particles (DSP) ${ }^{5,6}$ and macro-defect-free cements $(\mathrm{MDF})^{7.8}$ are especially impressive. The compressive strengths of these materials readily approach $150 \mathrm{MPa}$ or even higher. However, despite the ultra-high strength, these materials suffer from 'brittleness' which can lead to catastrophic failure without noticeable warning.

In parallel to the developments of high-strength concretes, efforts have been extended to exploring fiber reinforcement. ${ }^{9-11}$ Significant toughness 
improvements have been achieved with various types of fibers such as glass, steel, or polymers. Several orders of magnitude differences are generally found in toughness as reflected in the long post-peak tails of the tensile stress/deformation curves, which is largely attributed to fiber pullout. ${ }^{12.1 .3}$ Nevertheless, little or no improvement in strength or ductility was typically reported for low-fiber-loaded composites. This is perhaps due to improper combinations of material constituents, and inadequate fiber reinforcement. Furthermore, it is less clear what the intrinsic relationship between strength increase and ductility enhancement of random discontinuous fiber composites is. The full merits of fiber reinforcement can only be achieved if the individual roles played by fiber, matrix, and their interface are understood. Hence a micromechanics-based design methodology can provide guidelines for selection of individual components to satisfy one or several composite performance requirements. This is especially valid in design for tensile strength and ductility of advanced cementitious composites. These properties are typically not considered in structural design. Nevertheless, a new generation of cementitious composites with high strength as well as excellent ductility may be made based on our recent research.

In this paper, we address this issue from a micromechanics point of view, and highlight the implication of new findings. Experimental results of direct tensile tests of random discontinuous fiber-reinforced high-strength concretes and OPC paste are presented and discussed.

\section{EXPERIMENTAL PROCEDURE}

\subsection{Matrix}

Two types of commercially available preblended cementitious products were used. They contained fine aggregates, sand, silica fume, and special chemical admixtures, namely BKR (Elkem Materials, Norway) and WC1000 (Elkem Chemicals, USA). These products are considered highstrength concretes. In addition, ordinary type I Portland cement paste was also used as a weak matrix. The constituent materials used and their mix proportions are given in Table 1.

\subsection{Fiber}

A high-modulus polyethylene fiber (Spectra 900, Allied Corp) and steel fiber (Dramix, Bekaert Corp) were employed in this study. Properties of these fibers are tabulated in Table 2. Various fiber
Table 1. Mix proportions of matrices tested

\begin{tabular}{|c|c|c|c|}
\hline Name & $w / c^{\prime \prime}$ & $S F / C^{\prime \prime}$ & $S P / C^{*}$ \\
\hline OPC (type I) & $0 \cdot 27$ & $0 \cdot 2$ & 0.03 \\
\hline BKR & $0.06^{\prime \prime}$ & NA & NA \\
\hline WC 1000 & $0.065^{h}$ & NA & NA \\
\hline
\end{tabular}

"c = coment; $w=$ water; $S F=$ silica fume; $S P=$ superplasticizer.

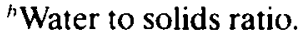

' $\mathrm{NA}=$ information not available to us.

Table 2. Properties of fibers used in this study

\begin{tabular}{lcccc}
\hline \multicolumn{1}{c}{ Type } & $\begin{array}{c}E_{i}{ }^{\prime \prime} \\
(G P a)\end{array}$ & $\begin{array}{c}\sigma_{1}{ }^{\prime \prime} \\
(G P a)\end{array}$ & $\begin{array}{c}d^{\prime \prime} \\
(\mathrm{mm})\end{array}$ & $\begin{array}{c}L_{+}{ }^{\prime \prime} \\
(\mathrm{mm})\end{array}$ \\
\hline Steel & 200 & $2 \cdot 8$ & $0 \cdot 15$ & 6 \\
Polyethylene & 120 & $2 \cdot 6$ & $0 \cdot 038$ & $12 \cdot 7$ \\
\hline
\end{tabular}

" $E_{1}=$ fiber modulus; $\sigma_{1}=$ fiber strength, $d_{1}=$ fiber diameter; $L_{\mathrm{f}}=$ fiber length.

volume fractions were used. Steel fibers were mixed with strong matrices of BKR and WC1000 only, whereas polyethylene fibers were used in both strong and weak matrices.

\subsection{Preparation and testing}

Direct tensile tests were performed on coupon specimens with dimensions of $12.7 \mathrm{~mm} \times 76.2$ $\mathrm{mm} \times 304.8 \mathrm{~mm}$. A regular Hobart mixer was used in mixing the materials. The specimens were cast in Plexiglas moulds immediately after mixing using a high-frequency vibration table. They were then allowed to harden at room temperature for two days prior to demoulding and subsequently cured in water for four weeks before testing. For OPC paste, specimens were demoulded after one day. In addition, compression tests were also conducted on $76 \cdot 2-\mathrm{mm} \times 152 \cdot 4-\mathrm{mm}$ cylindrical specimens as a supplement to direct tensile test.

The uniaxial tensile tests were conducted in a closed loop servo-hydraulic testing machine. The specimens were loaded to failure with a constant cross-head speed of $0.005 \mathrm{~mm} / \mathrm{s}$. Two linear variable differential transducers (LVDTs) were attached to opposite sides of the specimens to monitor continuously the strain changes at a gauge length of $205 \mathrm{~mm}$.

For the compression tests, a constant crosshead speed of $0.025 \mathrm{~mm} / \mathrm{s}$ was used.

\section{EXPERIMENTAL RESULTS}

\subsection{Strong matrix}

In Fig. 1, the BKR matrix shows impressive tensile strength, as high as $10.5 \mathrm{MPa}$. Following the 


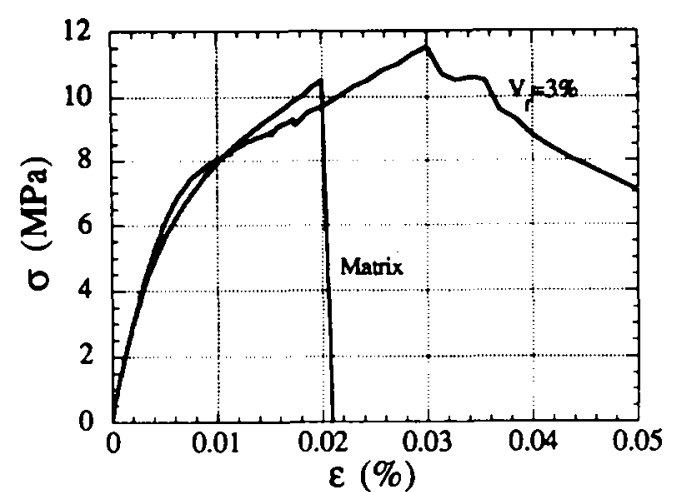

Fig. 1. Tensile stress/strain curves of stecl-fiber-reinforced BKR matrix.

peak load, a catastrophic failure occurs. On the same graph, 3\% steel fiber reinforcement enhances significantly only the fracture energy (as indicated by the area under the softening branch of the stress-deformation curve). It should be noted that the entire deformation of the specimen is completely attributed to continuous opening of the major crack after the peak load. Hence it is not 'actual' strain after formation of a first crack except in the case where multiple cracking occurs. There is little effect on composite ultimate strength $(-10 \%$ increase $)$ and strain $(\sim 50 \%$ increase), hence little improvement in ductility is noticed.

In the case of WC1000, although the base matrix is not as strong as the BKR, it is still much stronger (at 6.5 MPa tensile strength) than ordinary concrete. With $6 \%$ steel reinforcement, a $95 \%$ increase in composite ultimate strength and $250 \%$ increase in composite ultimate strain are observed. However, there are no further improvements when $V_{\mathrm{f}}$ increases to $8 \%$. For the latter case, strength and strain increases are $44 \%$ and $150 \%$ respectively. This may be due to the much poorer workability of the $8 \%$ fiber composites.

In a separate mix, high-modulus polyethylene fibers were used. For this series of tests, the water/ solid ratio $(\mathrm{w} / \mathrm{s})$ increased from $6.5 \%$ to $8 \%$. The plain matrix with no fibers exhibits tensile strength of $5.1 \mathrm{MPa}$ which is $22 \%$ less than that for the mix with a w/s $=6.5 \%$. With $2 \%$ polyethylene fibers, the ultimate strength and strain are slightly improved $(\sim 10 \%$ and $70 \%$ increase respectively), as shown in Fig. 3. The fiber-reinforced strong matrices in Figs 1-3 exhibit typical stress/strain curves of 'ordinary' FRC. The long tail-like descending portion is caused by fiber pull-out.

It should be noted that the effects of fiber reinforcement in either BKR or WC1000 are much

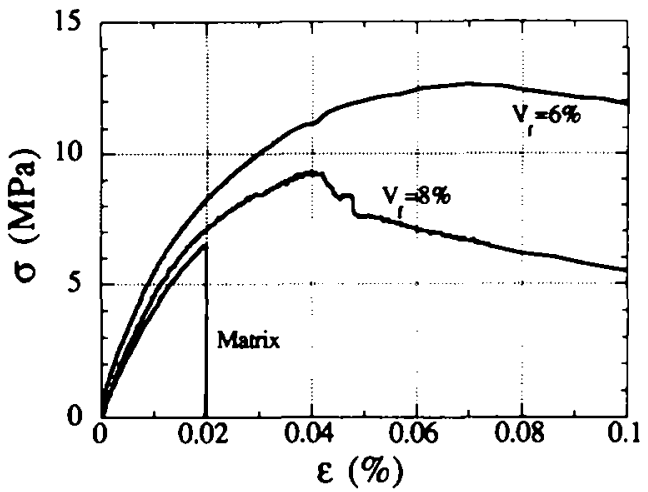

Fig. 2. Tensile stress/strain curves of steel-fiber-reinforced WC 1000 matrix.

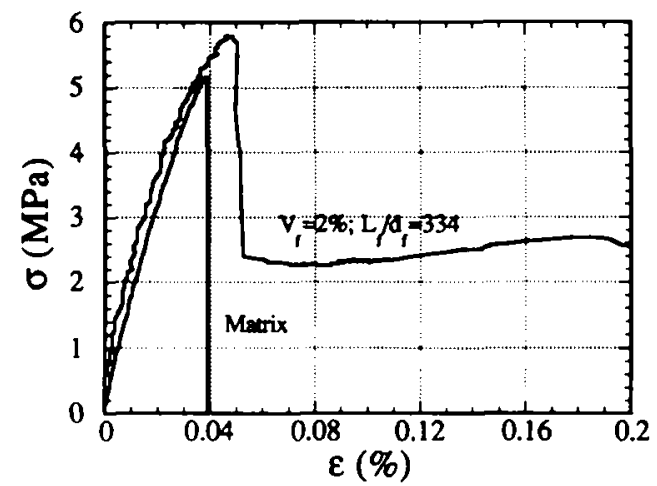

Fig. 3. Tensile stress/strain curves of polyethylene-fiberreinforced WC1000 matrix,

greater on ultimate strain capacity than on ultimate strength. This nonlinearity is probably due to microcrack development prior to the opening of one macrocrack. The exact cracking mechanisms deserve further investigation. In addition, different descending modes are observed for steel and polyethylene fiber composites (see Figs 1-3). In the polyethylene composites (Fig. 3), the load rises again after a sudden drop; this corresponds to the opening of a single crack. The bridging fibers can still bear the composite loads until the maximum bridging stress is reached. By contrast, if the maximum bridging stress has been exceeded during the propagation of the single crack, the second peak disappears as shown in Figs 1 and 2 . Detailed discussion on these failure modes can be found in $\mathrm{Li}$ and Leung. ${ }^{10}$

The compressive strengths of BKR and WC1000 are $140 \mathrm{MPa}$ and $75 \mathrm{MPa}$ respectively after 28 days curing in water.

\subsection{Weak matrix}

The high-modulus polyethylene fibers were mixed with OPC paste $(w / c=0 \cdot 27)$. Two significantly different failure modes are found for these composites, as shown in Fig. 4. For the cases of 


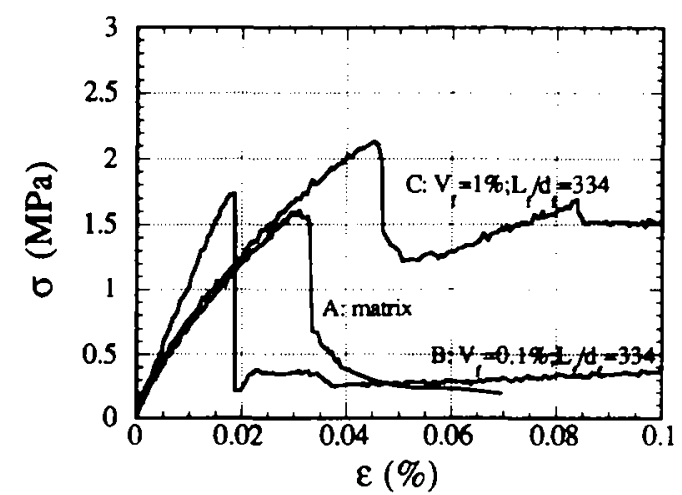

(a)

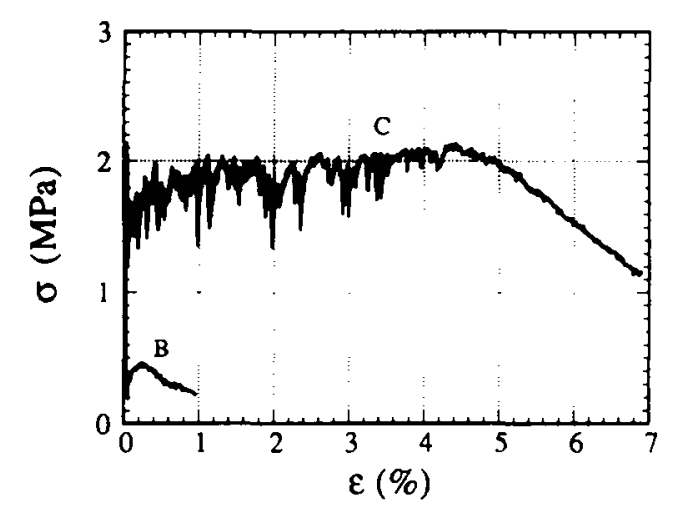

(b)

Fig. 4. Tensile stress/strain curves of polyethylene-fiberreinforced OPC paste: (a) initial portions, (b) complete curves.

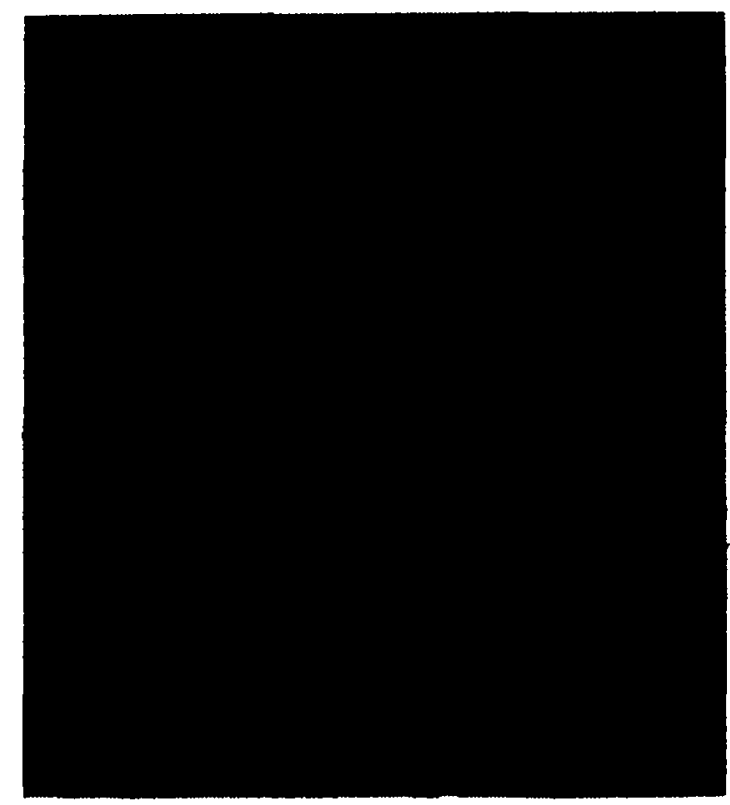

Fig. 5. Spectra fiber-reinforced OPC paste $\left(V_{\mathrm{f}}=1 \%\right)$ showing multiple cracking.

$V_{\mathrm{f}}=0.1 \%$ and $1 \%$, the corresponding failure strains are $0.02 \%$ and $4.5 \%$. The mix with $0.1 \%$ fiber shows typical tensile behavior with a single macrocrack opening with descending loads. The addition of $1 \%$ fiber significantly enhanced ductility as well as providing enormous fracture energy. Clear subparallel multiple cracking was observed in this 'ductile' composite (see Fig. 5). During multiple cracking, it was found that the load was more or less maintained and may even increase slightly, as expected for a pseudo strainhardening material. ${ }^{10.19}$ On the same graph, the matrix alone, showing catastrophic failure, is also included for comparison.

The 28-day compressive strength of the OPC paste is $20 \mathrm{MPa}$. Some visible shrinkage cracks were observed on the surface of the compressive strength cylinder specimens. These cracks were not found on the tensile specimens. This difference is probably caused by a much more severe shrinkage restraint imposed on the cylinders.

\section{DISCUSSION}

\subsection{Strength and pseudo ductility}

The strength of monolithic materials is generally considered as a material intrinsic property which is dependent on the nature of the bonding elements on a microscopic level. Covalent bonding is much stronger than ionic or hydrogen bonding. Van der Waal's forces are the weakest. In addition, the intrinsic flaw size also limits material strength. ${ }^{14}$ Pre-existing flaws are preferential sites of crack initiation and propagation. Hence any mechanisms which impede crack propagation are beneficial to strength improvement. Microcrack suppression due to fibers is believed to be the major strengthening mechanism in fiber-reinforced composites. This is true for both aligned continuous and random discontinuous fiber composites. As for pseudo ductility of the cementitious composites, it results from a multiple cracking phenomenon. ${ }^{15-17}$ The composites will undergo pseudo strain-hardening when loaded beyond the elastic limit. This means that beyond the limit of proportionality, the new cement composite behaves more like ductile metals than ordinary concretes which are more brittle in nature, or ordinary FRCs which are quasi-brittle. ${ }^{18}$

The pseudo strain-hardening process is made possible by the stress transfer capability of bridging fibers. This phenomenon is associated with the multiple cracking of the brittle matrix. When the composite is loaded up to its first cracking strength under uniaxial tensile loading, a first macroscopic crack is formed in the composite. The composite load is shared by the bridging 
fibers. These fibers then transfer the load via their interface back into the matrix. If enough load is transferred, the matrix may crack again and the process repeats until the matrix is broken by a series of subparallel cracks of approximately equal crack spacing. ${ }^{19}$ Straining of the bridging fibers across the matrix cracks and within the matrix blocks gives rise to a composite strain that can be substantially higher than the matrix failure strain alone. The importance of this enhanced strain capacity is due to the maintained or even rising composite load during this straining process.

The pseudo strain-hardening process and related composite tensile properties have been addressed by a number of investigators (see e.g. Refs 15-17 for aligned continuous and Refs 10 and 19 for random discontinuous fiber-reinforced composites). These models provide quantitative information on the linkage between the characteristics of material constituents and the composite properties. This is of importance in tailoring material components (i.e. mechanical and geometric properties of fiber, matrix, and their interface) to achieve multiple cracking.

\subsection{Conditions for multiple cracking}

The present micromechanical analysis of random discontinuous fiber-reinforced composites is based on a fracture mechanics approach whereby the bridging action of fibers is treated as cohesive traction acting across the crack flanks. ${ }^{10}$ In deriving such a relationship, fiber randomness of both location and orientation is explicitly considered, expressed as probability distribution functions. ${ }^{11}$ In addition, the snubbing effect which amplifies the bridging force due to fiber/matrix snubbing when a fiber is pulled out at an angle to the loading axis, is also accounted for in the snubbing factor, $g .^{20}$

Conditions for steady-state cracking and multiple cracking are found to depend on two nondimensional parameters which embody all relevant material micromechanical parameters. ${ }^{11}$ Alternatively, a critical fiber volume fraction, $V_{\mathrm{f}}^{\mathrm{cri}}$, can be defined as the minimum fiber quantity required for achieving multiple cracking. ${ }^{10,19}$ This leads to

$$
V_{\mathrm{f}} \geq V_{\mathrm{f}}^{\text {crit }} \equiv 48 G_{\text {tip }} /\left[g \tau d_{\mathrm{f}}\left(L_{\mathrm{f}} / d_{\mathrm{f}}\right)^{2} \delta^{*}\right]
$$

where $G_{\mathrm{tip}}=$ crack-tip fracture-energy-absorption rate; $g=$ snubbing factor; $\tau=$ bond strength; $d_{\mathrm{f}}=$ fiber diameter; $L_{\mathrm{f}}=$ fiber length; $\delta^{*}=[2 \tau]$ $\left.(1+\eta) E_{\mathrm{f}}\right]\left(L_{\mathrm{f}} / d_{\mathrm{f}}\right) ; \quad \eta=V_{\mathrm{f}} E_{\mathrm{f}} / V_{\mathrm{m}} E_{\mathrm{m}} ; \quad E=$ modulus;
$V=$ volume fraction; and subscripts $\mathrm{f}$ and $\mathrm{m}$ refer to fiber and matrix respectively.

Note that (1) clearly reflects the importance of the crack tip and therefore the matrix toughness in the condition for pseudo strain-hardening. The required fiber volume fraction increases for a matrix of higher fracture toughness.

However, a real solution is not always guaranteed from (1) because the fiber bridging fracture energy (associated with the ascending branch of the bridging stress vs. crack opening curve) does not increase monotonically with $V_{\mathrm{f}}{ }^{19}$ Therefore, energy-absorption rate in the fiber-bridging zone behind the crack front cannot keep up with cracktip fracture-energy-absorption rate. Hence, the auxiliary condition can be obtained by requiring (1) to yield a real solution for $V_{\mathrm{f}}$, leading to

$$
\tau^{2}\left(L_{\mathrm{f}} / d_{\mathrm{f}}\right)^{3} \geq \frac{24\left(1-v^{2}\right) E_{\mathrm{f}}(1+\eta) K_{\mathrm{m}}^{2} E_{\mathrm{c}}}{E_{\mathrm{m}}^{2} d_{\mathrm{f}} g V_{\mathrm{f}}^{\prime}}
$$

where $\nu=$ Poisson ratio; $K_{\mathrm{m}}=$ fracture toughness of matrix; and $V_{\mathrm{f}}^{\prime}$ must satisfy the following equation

$$
\begin{gathered}
V_{\mathrm{f}}^{\prime}\left[E_{\mathrm{c}}\left(E_{\mathrm{f}}-E_{\mathrm{m}}\right)+(1+\eta) V_{\mathrm{m}}^{\prime} E_{\mathrm{m}} \frac{\partial E_{\mathrm{c}}}{\partial V_{\mathrm{f}}^{\prime}}\right] \\
=(1+\eta) E_{\mathrm{m}} E_{\mathrm{c}}\left(1-2 V_{\mathrm{f}}^{\prime}\right)
\end{gathered}
$$

Equation (2) puts a lower limit on fiber aspect ratio and interface bond. Hence increasing $V_{f}$ does not guarantee pseudo strain-hardening, and may lead to processing difficulties.

\subsection{Problems related to suppression of pseudo ductility}

As revealed in (1), $V_{f}^{\text {crit }}$ depends on matrix toughness, fiber aspect ratio, and fiber/matrix bond strength, provided that the auxiliary condition, eqn (2), is satisfied. Low matrix toughness and high fiber aspect ratio are in favour of low $V_{\mathrm{f}}^{\mathrm{crt}}$. Unfortunately, a strong matrix is generally associated with high toughness. ${ }^{21}$ Further, a denser packing leading to smaller flaw size is typically observed for a strong matrix. Hence the first cracking strength may readily exceed the maximum bridging stress so that the pseudo strainhardening behavior is suppressed. Therefore, in a low toughness matrix (or weak matrix) it is relatively easy to achieve multiple cracking, albeit with a lower composite strength. This prediction is confirmed in our experiments. The fracture toughness of the strong matrix (BKR) and weak 
Table 3. Fiber, matrix and interface propertics used in calculation of $V_{i}^{i r l}$

\begin{tabular}{|c|c|c|c|c|c|c|c|}
\hline Matrix & $\begin{array}{c}E_{\mathrm{m}} \\
(\mathrm{GPa})\end{array}$ & $\begin{array}{c}K_{(m)} \\
(\mathrm{MPa} \sqrt{\mathrm{m}})\end{array}$ & $v$ & Fiber & $g$ & $\stackrel{T^{\prime \prime}}{\mathrm{MPa}}$ & $\begin{array}{l}v_{i}^{\prime \prime \prime} \\
\cdots, 1\end{array}$ \\
\hline BKR & 40 & $2 \cdot 0$ & 0.2 & Steel & 2 & 0 & $\ldots$ \\
\hline WC1000 & 40 & $2 \cdot()^{a}$ & $0 \cdot 2$ & Steel & 2 & 6 & $-n$ \\
\hline WC 1000 & 40 & $2 \cdot 0^{a}$ & $0 \cdot 2$ & Polyethylene & 2 & 1.5 & $7 \cdot 8$ \\
\hline OPC & 15 & 0.3 & $0 \cdot 2$ & Polyethylenc & 2 & $1 \cdot 0$ & 0.8 \\
\hline
\end{tabular}

"Estimated

"In violation of eqn (2).

OPC paste used in this study are determined to be $2.0 \mathrm{MPa} \sqrt{\mathrm{m}}$ and $0.3 \mathrm{MPa} \sqrt{\mathrm{m}}$ respectively from fracture tests. ${ }^{22}$ Hence, $V_{\mathrm{f}}^{\text {crit }}$ predicted from (1) is $7.8 \%$ for polyethylene fibers in the strong matrix. As for steel fibers, the auxiliary condition (2) is found not to be satisfied owing to low fiber aspect ratio. Therefore, for a strong matrix with both fibers, good strength with no pseudo ductility is expected, as shown in Figs 1-3. For the weak matrix with polyethylene fibers, $V_{i}^{\text {crit is calculated }}$ to be as low as $0.8 \%$. Not surprisingly, two significantly different failure modes are found, as shown in Fig. 4. The ultimate failure strain for the composite with pseudo strain-hardening (with

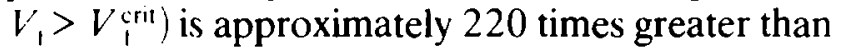
that of the mix without strain-hardening $\left(V_{\mathrm{f}}<\right.$ $\left.V_{\mathrm{f}}^{\mathrm{cnt}}\right)$. Other parameters used in calculation of $V_{\mathrm{f}}^{\text {cri }}$ are tabulated in Table 3.

In practice, $V_{\text {, }}$ is limited by mixing difficulty. High-fiber-loaded composites are notorious for their poor workability and induced voids (hence low bond strength). Proper combinations of matrix toughness, fiber aspect ratio, and bond strength can result in adequate $V_{\mathrm{i}}^{\mathrm{crit}}$. High bond strength can be used to reduce $V_{f}^{\text {cirt }}$ and limiting $\left(L_{t} / d_{1}\right)$ as required by $(2)$. However, when bond strength gets too high, fiber rupture may occur and lead to a brittle failure. ${ }^{23}$ Hence an optimum composite design should include properly tailored bond strength so that minimum fiber quantity can ensure desired composite performance at a minimum cost.

\section{CONCLUSIONS}

In this paper, the mechnanisms responsible for strength and ductility improvements of random discontinuous fiber-reinforced cementitious composites are discussed from a micromechanics point of view. In particular, the conditions for multiple cracking are reviewed. A critical fiber volume fraction, $V_{\mathrm{f}}^{\text {crit }}$, is found to govern the occurrence of multiple cracking, provided that the auxiliary condition, (2), is satisfied. Low matrix toughness, high fiber aspect ratio, and high interfacial bond strength are in favor of low $V_{\mathrm{f}}^{\text {crit }}$. However, a strong matrix is generally associated with high fracture toughness. It is difficult to make a composite strong as well as ductile, unless other micromechanical parameters as mentioned in this paper can be adjusted so that an adequate $V_{\mathrm{r}}^{\text {crit is }}$ achieved. These concepts are demonstrated experimentally with several combinations of matrices and fiber types.

\section{ACKNOWLEDGEMENTS}

The authors acknowledge helpful discussions with T. Hashida and M. Maalej, who provided the fracture toughness data for the various matrices used in this study. The authors also thank Y. Chen for preparing some of the specimens. Funding from the National Science Foundation to the ACEMRL at the University of Michigan is gratefully acknowledged.

\section{REFERENCES}

1. Mindess, S. \& Young, F., Concrete. Prentice Hall, 1981.

2. National Research Council (1987) Concrete Durability: A Multibillion-dollar Opportunity. Performed by National Materials Advisory Board of NRC. NMAB437.

3. Gouda. G. R. \& Roy, D. M.. Cem. Concr. Res.. 5 (1975) 551

4. Roy, D. M., Advances in Cements/Chemically Bonded Ceramics, Centennial of the Ceramic Society of Japan. Tokyo (1991).

5. Bache, H. H., Densified cement/ultrafine particle-based materials. In Proc. 2nd Int. Conf. on Superplasticizers in Concrete, Ottawa (1981) p. 33.

6. Bache, H. H., High strength concrete development through 25 years. Reprint No. 17, presented at Dansk Betondag, 13 Nov. 1987.

7. Birchall, J. D., Howard, A. J. \& Kendall, K., Europe. Pat. Publ. No 0021682 (1982). 
8. Birchall, J. D., Cement in the context of new materials for an energy-expensive future. Phil. Trans. $R$. Soc. Lond., A310 (1983) 31-42.

9. Shah, S. P., Toughening of cement-based materials with fiber reinforcement. Mat. Res. Sym. Proc., 211 (1991) 3-13.

10. Li, V. C. \& Leung, C. K. Y., Theory of steady state and multiple cracking of random discontinuous fiber reinforced brittle matrix composites. ASCE J. Engng Mechanics, 118 (1992) 2246-64.

11. Li, V. C., Wang, Y. \& Backer, S., A micromechanical model of tension-softening and bridging toughening of short random fiber reinforced brittle matrix composites. J. Mech. Phys. Solids, 39 (1991) 607-25.

12. Wang, Y., Li, V. C. \& Backer, S., Tensile failure mechanisms in synthetic fiber-reinforced mortar. J. Mater. Sci., 26 (1991) 6565-75.

13. Li, V. C., Wang, Y.\& Backer, S., Fracture energy optimization in synthetic fiber reinforced cementitious composites. Mat. Res. Soc. Symp. Proc., 211 (1991) 63-9.

14. Griffith. A. A.. The phenomena of rupture and flow in solids. Phil. Trans. Roy. Soc. Lond., A221 (1921) 163-97.

15. Aveston, J., Cooper, G. A. \& Kelly, A., Single and multiple fracture. In The Properties of Fiber Composites. Conf. Proc., IPC Sci. \& Technology Press Ltd, 1971, pp. 15-24.

16. Marshall, D. B., Cox, B. N. \& Evans, A. G.. The mechanics of matrix cracking in brittle-matrix fiber composites. Acta Metall., 33 (1985) 2013-21.

17. Marshall, D. B. \& Cox, B. N., Tensile fracture of brittle matrix composites: Influence of fiber strength. Acta Metall., 35 (1987) 2607-19.

18. Li, V. C. \& Hashida, T., Engineering ductile fracture in brittle-matrix composites. J. Mater. Sci. Lett., 12 (1993) 898-901.

19. Li, V. C. \& Wu, H. C., Conditions for pseudo strainhardening in fiber reinforced brittle matrix composites. Applied Mechanics Reviews, 45 (1992) 390-8.

20. Li, V. C., Wang, Y.\& Backer, S., Effect of inclining angle, bundling and surface treatment on synthetic fiber pullout from a cement matrix. Composites, 21 (1990) $132-40$.

21. Wu, H. C.. Mishra, D. K. \& Li, V. C., Influence of matrix fracture toughness on tensile behaviour of fiber reinforced cementitious composites. To appear in Proc. 3rd Beijing Int'l Symp. on Cement and Concrete, Beijing, PRC, 1993.

22. Maalej, M., Fracture resistance of engineered fiber cementitious composites and implications to structural behavior. PhD Thesis, Department of Civil and Environmental Engineering, The University of Michigan, 1994.

23. Maalej, M., Li, V. C. \& Hashida, T., Effect of fiber rupture on tensile properties of short fiber composites. ASCE J. Eng. Mech. (1993) submitted. 